ANALYSIS \& PDE

Volume $9 \quad$ No. $4 \quad 2016$

HUICHI HUANG

MEAN ERGODIC THEOREM FOR AMENABLE DISCRETE QUANTUM GROUPS AND A WIENER-TYPE THEOREM FOR COMPACT METRIZABLE GROUPS 


\title{
MEAN ERGODIC THEOREM FOR AMENABLE DISCRETE QUANTUM GROUPS AND A WIENER-TYPE THEOREM FOR COMPACT METRIZABLE GROUPS
}

\author{
HUICHI HUANG
}

We prove a mean ergodic theorem for amenable discrete quantum groups. As an application, we prove a Wiener-type theorem for continuous measures on compact metrizable groups.

1. Introduction $\quad 893$

2. Preliminaries $\quad 896$

3. Mean ergodic theorem for amenable discrete quantum groups 899

4. A Wiener-type theorem for compact metrizable groups 903

Acknowledgements 905

$\begin{array}{ll}\text { References } & 906\end{array}$

\section{Introduction}

A countable discrete group $\Gamma$ is called amenable if there exists a sequence $\left\{F_{n}\right\}_{n=1}^{\infty}$ (called a right Følner sequence) consisting of finite subsets $F_{n}$ of $\Gamma$ such that

$$
\lim _{n \rightarrow \infty} \frac{1}{\left|F_{n}\right|}\left|F_{n} s \Delta F_{n}\right|=0
$$

for every $s \in \Gamma$.

Let $(X, \mathscr{B}, \mu, \Gamma)$ be a dynamical system consisting of a countable discrete amenable group $\Gamma$ with a measure-preserving action on a probability space $(X, \mathscr{B}, \mu)$.

Recall that von Neumann's mean ergodic theorem for amenable group actions on measure spaces says the following:

Theorem 1.1 (measure space version of von Neumann's mean ergodic theorem [Glasner 2003, Theorem 3.33]). Let $\left\{F_{n}\right\}_{n=1}^{\infty}$ be a right Folner sequence of $\Gamma$. Then, for every $f \in L^{2}(X, \mu)$, the sequence $\left(1 /\left|F_{n}\right|\right) \sum_{s \in F_{n}} s \cdot f$ converges to $P f$ with respect to the $L^{2}$ norm, where $P$ is the orthogonal projection from $L^{2}(X, \mu)$ onto the space $\left\{g \in L^{2}(X, \mu) \mid s \cdot g=g\right.$ for all $\left.s \in \Gamma\right\}$.

R. Duvenhage [2008, Theorem 3.1] proves a generalization of von Neumann's mean ergodic theorem for coactions of amenable quantum groups on von Neumann algebras (noncommutative measure spaces). Later, a more general version was proved by V. Runge and A. Viselter [2014, Theorem 2.2].

Supported by ERC Advanced Grant No. 267079.

MSC2010: 37A30, 43A05, 46L65.

Keywords: mean ergodic theorem, coamenable compact quantum group, amenable discrete quantum group, continuous measure. 
There is also a version of von Neumann's mean ergodic theorem for amenable group actions on Hilbert spaces, which says the following:

Theorem 1.2 (Hilbert space version of von Neumann's mean ergodic theorem). Let $\left\{F_{n}\right\}_{n=1}^{\infty}$ be a right Folner sequence of a countable discrete amenable group $\Gamma$ and $\pi: \Gamma \rightarrow B(H)$ be a unitary representation of $\Gamma$ on a Hilbert space $H$. Set $H_{\Gamma}=\{x \in H \mid \pi(s) x=x$ for all $s \in \Gamma\}$. Then

$$
\lim _{n \rightarrow \infty} \frac{1}{\left|F_{n}\right|} \sum_{s \in F_{n}} \pi(s)=P
$$

under the strong operator topology on $B(H)$, where $P$ is the orthogonal projection from $H$ onto $H_{\Gamma}$.

The group $C^{*}$-algebra $C^{*}(\Gamma)$ equals $C(G)$ for a coamenable compact quantum group $G$ with the dual group $\widehat{G}=\Gamma$. The counit $\varepsilon$ of $G$ is given by $\varepsilon\left(\delta_{s}\right)=1$ for all $s \in \Gamma$. Hence,

$$
H_{\Gamma}=\left\{x \in H \mid \pi(a) x=\varepsilon(a) x \text { for all } a \in C^{*}(\Gamma)\right\} .
$$

With these in mind, the Hilbert space version of von Neumann's mean ergodic theorem can be reformulated in the framework of compact quantum groups as follows.

Suppose $G$ is a coamenable compact quantum group such that the dual $\widehat{G}$ is a countable discrete amenable group $\Gamma$. Let $\left\{F_{n}\right\}_{n=1}^{\infty}$ be a right Følner sequence of $\Gamma$ and $\pi: C(G)=C^{*}(\Gamma) \rightarrow B(H)$ be a representation of $C^{*}(\Gamma)$ on a Hilbert space $H$. Then

$$
\lim _{n \rightarrow \infty} \frac{1}{\left|F_{n}\right|} \sum_{s \in F_{n}} \pi(s)=P
$$

under the strong operator topology on $B(H)$, where $P$ is the orthogonal projection from $H$ onto $H_{\Gamma}=$ $\left\{x \in H \mid \pi(a) x=\varepsilon(a) x\right.$ for all $\left.a \in C^{*}(\Gamma)\right\}$.

D. Kyed proves that a compact quantum group $G$ is coamenable if and only if there exists a right Følner sequence $\left\{F_{n}\right\}_{n=1}^{\infty}$ of finite subsets in its dual $\widehat{G}$, that is to say, $G$ is a coamenable compact quantum group if and only if $\widehat{G}$ is an amenable discrete quantum group [2008, Definition 4.9.]. ${ }^{1}$ So it is natural to ask for a generalization of the Hilbert space version of von Neumann's mean ergodic theorem to all amenable discrete quantum groups. This is the main result of the paper.

Theorem 3.1 (mean ergodic theorem for amenable discrete quantum groups). Let $G$ be a coamenable compact quantum group with counit $\varepsilon$ and let $\left\{F_{n}\right\}_{n=1}^{\infty}$ be a right Folner sequence of $\widehat{G}$. Set $H_{\mathrm{inv}}=$ $\{x \in H \mid \pi(a) x=\varepsilon(a) x$ for all $a \in A\}$. For a representation $\pi: A=C(G) \rightarrow B(H)$, we have

$$
\lim _{n \rightarrow \infty} \frac{1}{\left|F_{n}\right|_{w}} \sum_{\alpha \in F_{n}} d_{\alpha} \pi(\chi(\alpha))=P
$$

under the strong operator topology, where $P$ is the orthogonal projection from $H$ onto $H_{\mathrm{inv}}$.

\footnotetext{
${ }^{1}$ The existence of a Følner sequence for Kac-type compact quantum groups is shown by Z. Ruan [1996]. Also see [Tomatsu 2006].
} 
Here $\left|F_{n}\right|_{w}$ stands for the weighted cardinality of $F_{n}$. Definitions of $\left|F_{n}\right|_{w}, d_{\alpha}$ and $\chi(\alpha)$ are in Section 2. The left-hand side of (1-1) involves both a representation of a coamenable compact quantum group $G$ and that of its discrete quantum group dual $\widehat{G}$, so it illustrates some interactions between them.

The rest of the paper aims at an application of Theorem 3.1. Namely, we prove a Wiener-type theorem for finite Borel measures on compact metrizable groups.

A finite Borel measure $\mu$ on a compact metrizable space $X$ is called continuous or nonatomic if $\mu\{x\}=0$ for every $x \in X$.

The following theorem of N. Wiener [1933] expresses finite Borel measures on the unit circle via their Fourier coefficients.

Theorem 1.3 (Wiener's theorem [Katznelson 2004, Chapter 1, Theorem 7.13]). For a finite Borel measure $\mu$ on the unit circle $\mathbb{T}$ and every $z \in \mathbb{T}$, one has

$$
\lim _{N \rightarrow \infty} \frac{1}{2 N+1} \sum_{n=-N}^{N} \hat{\mu}(n) z^{-n}=\mu\{z\} \quad \text { and } \quad \lim _{N \rightarrow \infty} \frac{1}{2 N+1} \sum_{n=-N}^{N}|\hat{\mu}(n)|^{2}=\sum_{x \in \mathbb{T}} \mu\{x\}^{2} .
$$

Hence, $\mu$ is continuous if and only if

$$
\lim _{N \rightarrow \infty} \frac{1}{2 N+1} \sum_{n=-N}^{N}|\hat{\mu}(n)|^{2}=0,
$$

where $\hat{\mu}(n):=\int_{\mathbb{T}} z^{n} d \mu(z)$ for $n \in \mathbb{Z}$ are the Fourier coefficients of $\mu$.

There are various generalized Wiener's theorems (we call such generalizations Wiener-type theorems), including a version for compact manifolds [Taylor 1981, Chapter XII, Theorem 5.1], a version for compact Lie groups by M. Anoussis and A. Bisbas [2000, Theorem 7], and a version for compact homogeneous manifolds by M. Björklund and A. Fish [2009, Lemma 2.1].

We apply the above mean ergodic theorem (Theorem 3.1) to get a Wiener-type theorem on compact metrizable groups. This version differs from previous ones mainly in two aspects: firstly we don't require smoothness on spaces; secondly we use a different Følner condition.

Theorem 4.1 (Wiener-type theorem for compact metrizable groups). Let $G$ be a compact metrizable group. Given $y$ in $G$ and a right Folner sequence $\left\{F_{n}\right\}_{n=1}^{\infty}$ of $\widehat{G}$, for a finite Borel measure $\mu$ on $G$ one has $\lim _{n \rightarrow \infty} \frac{1}{\left|F_{n}\right|_{w}} \sum_{\alpha \in F_{n}} d_{\alpha} \sum_{1 \leq i, j \leq d_{\alpha}} \mu\left(u_{i j}^{\alpha}\right) \overline{u_{i j}^{\alpha}(y)}=\mu\{y\} \quad$ and $\quad \lim _{n \rightarrow \infty} \frac{1}{\left|F_{n}\right|_{w}} \sum_{\alpha \in F_{n}} d_{\alpha} \sum_{1 \leq i, j \leq d_{\alpha}}\left|\mu\left(u_{i j}^{\alpha}\right)\right|^{2}=\sum_{x \in G} \mu\{x\}^{2}$. Hence, $\mu$ is continuous if and only if

$$
\lim _{n \rightarrow \infty} \frac{1}{\left|F_{n}\right|_{w}} \sum_{\alpha \in F_{n}} d_{\alpha} \sum_{1 \leq i, j \leq d_{\alpha}}\left|\mu\left(u_{i j}^{\alpha}\right)\right|^{2}=0 .
$$

Here the $u_{i j}^{\alpha}$ are the matrix coefficients of the irreducible unitary representation $\alpha$ of $G$; see Section 2 for the precise definition.

The paper is organized as follows. 
In Section 2, we collect some basic facts in compact quantum group theory. In Section 3, we prove the mean ergodic theorem, i.e., Theorem 3.1. As a consequence, we obtain Corollary 3.7, which is used in Section 4 to prove Theorem 4.1 .

\section{Preliminaries}

Conventions. Within this paper, we use $B(H, K)$ to denote the space of bounded linear operators from a Hilbert space $H$ to another Hilbert space $K$, and $B(H)$ stands for $B(H, H)$.

A net $\left\{T_{\lambda}\right\} \subset B(H)$ converges to $T \in B(H)$ under the strong operator topology (SOT) if $T_{\lambda} x \rightarrow T x$ for every $x \in H$, and $\left\{T_{\lambda}\right\}$ converges to $T \in B(H)$ under the weak operator topology (WOT) if $\left\langle T_{\lambda} x, y\right\rangle \rightarrow\langle T x, y\rangle$ for all $x, y \in H$.

The notation $A \otimes B$ always means the minimal tensor product of two $C^{*}$-algebras $A$ and $B$.

For a state $\varphi$ on a unital $C^{*}$-algebra $A$, we use $L^{2}(A, \varphi)$ to denote the Hilbert space of GelfandNeimark-Segal (GNS) representations of $A$ with respect to $\varphi$. The image of $a \in A$ in $L^{2}(A, \varphi)$ is denoted by $\hat{a}$.

In this paper all $C^{*}$-algebras are assumed to be unital and separable.

Some facts about compact quantum groups. Compact quantum groups are noncommutative analogues of compact groups. They were introduced by S. L. Woronowicz [1987; 1998].

Definition 2.1. A compact quantum group is a pair $(A, \Delta)$ consisting of a unital $C^{*}$-algebra $A$ and a unital $*$-homomorphism

$$
\Delta: A \rightarrow A \otimes A
$$

such that

(1) $(\mathrm{id} \otimes \Delta) \Delta=(\Delta \otimes \mathrm{id}) \Delta$;

(2) $\Delta(A)(1 \otimes A)$ and $\Delta(A)(A \otimes 1)$ are dense in $A \otimes A$.

One may think of $A$ as $C(G)$, the $C^{*}$-algebra of continuous functions on a compact quantum space $G$ with a quantum group structure. In the rest of the paper we write a compact quantum group $(A, \Delta)$ as $G$. The $*$-homomorphism $\Delta$ is called the coproduct of $G$.

There exists a unique state $h$ on $A$ such that

$$
(h \otimes \mathrm{id}) \Delta(a)=(\mathrm{id} \otimes h) \Delta(a)=h(a) 1_{A}
$$

for all $a$ in $A$. The state $h$ is called the Haar measure of $G$. Throughout this paper, we use $h$ to denote it.

For a compact quantum group $G$, there is a unique dense unital $*$-subalgebra $\mathscr{A}$ of $A$ such that:

(1) $\Delta$ maps from $\mathscr{A}$ to $\mathscr{A} \odot \mathscr{A}$ (the algebraic tensor product).

(2) There exists a unique multiplicative linear functional $\varepsilon: \mathscr{A} \rightarrow \mathbb{C}$ and a linear map $\kappa: \mathscr{A} \rightarrow \mathscr{A}$ such that $(\varepsilon \otimes \mathrm{id}) \Delta(a)=(\mathrm{id} \otimes \varepsilon) \Delta(a)=a$ and $m(\kappa \otimes \mathrm{id}) \Delta(a)=m(\mathrm{id} \otimes \kappa) \Delta(a)=\varepsilon(a) 1$ for all $a \in \mathscr{A}$, where $m: \mathscr{A} \odot \mathscr{A} \rightarrow \mathscr{A}$ is the multiplication map. The functional $\varepsilon$ is called the counit and $\kappa$ the coinverse of $C(G)$. 
Note that $\varepsilon$ is only densely defined and not necessarily bounded. If $\varepsilon$ is bounded and $h$ is faithful ( $h\left(a^{*} a\right)=0$ implies $a=0$ ), then $G$ is called coamenable [Bédos et al. 2001]. Examples of coamenable compact quantum groups include $C(G)$ for a compact group $G$ and $C^{*}(\Gamma)$ for a discrete amenable group $\Gamma$.

A nondegenerate (unitary) representation $U$ of a compact quantum group $G$ is an invertible (unitary) element in $M(K(H) \otimes A)$ for some Hilbert space $H$ satisfying that $U_{12} U_{13}=(\operatorname{id} \otimes \Delta) U$. Here $K(H)$ is the $C^{*}$-algebra of compact operators on $H$ and $M(K(H) \otimes A)$ is the multiplier $C^{*}$-algebra of $K(H) \otimes A$.

We write $U_{12}$ and $U_{13}$, respectively, for the images of $U$ by two maps from $M(K(H) \otimes A)$ to $M(K(H) \otimes A \otimes A)$, where the first one is obtained by extending the map $x \mapsto x \otimes 1$ from $K(H) \otimes A$ to $K(H) \otimes A \otimes A$, and the second one is obtained by composing this map with the flip on the last two factors. The Hilbert space $H$ is called the carrier Hilbert space of $U$. From now on, we always assume representations are nondegenerate. If the carrier Hilbert space $H$ is of finite dimension, then $U$ is called a finite-dimensional representation of $G$.

For two representations $U_{1}$ and $U_{2}$ with the carrier Hilbert spaces $H_{1}$ and $H_{2}$, respectively, the set of intertwiners between $U_{1}$ and $U_{2}, \operatorname{Mor}\left(U_{1}, U_{2}\right)$, is defined by

$$
\operatorname{Mor}\left(U_{1}, U_{2}\right)=\left\{T \in B\left(H_{1}, H_{2}\right) \mid(T \otimes 1) U_{1}=U_{2}(T \otimes 1)\right\} .
$$

Two representations $U_{1}$ and $U_{2}$ are equivalent if there exists a bijection $T$ in $\operatorname{Mor}\left(U_{1}, U_{2}\right)$. A representation $U$ is called irreducible if $\operatorname{Mor}(U, U) \cong \mathbb{C}$.

Moreover, we have the following well-established facts about representations of compact quantum groups:

(1) Every finite-dimensional representation is equivalent to a unitary representation.

(2) Every irreducible representation is finite-dimensional.

Let $\widehat{G}$ be the set of equivalence classes of irreducible unitary representations of $G$. For every $\gamma \in \widehat{G}$, let $U^{\gamma} \in \gamma$ be unitary and $H_{\gamma}$ be its carrier Hilbert space with dimension $d_{\gamma}$. After fixing an orthonormal basis of $H_{\gamma}$, we can write $U^{\gamma}$ as $\left(u_{i j}^{\gamma}\right)_{1 \leq i, j \leq d_{\gamma}}$ with $u_{i j}^{\gamma} \in A$, and

$$
\Delta\left(u_{i j}^{\gamma}\right)=\sum_{k=1}^{d_{\gamma}} u_{i k}^{\gamma} \otimes u_{k j}^{\gamma}
$$

for all $1 \leq i, j \leq d_{\gamma}$.

The matrix $\overline{U^{\gamma}}$ is still an irreducible representation (not necessarily unitary) with the carrier Hilbert space $\bar{H}_{\gamma}$. It is called the conjugate representation of $U^{\gamma}$ and the equivalence class of $\overline{U \gamma}$ is denoted by $\bar{\gamma}$.

Given two finite-dimensional representations $\alpha$ and $\beta$ of $G$, fix orthonormal bases for $\alpha$ and $\beta$ and write $\alpha$ and $\beta$ as $U^{\alpha}$ and $U^{\beta}$ in matrix forms, respectively. Define the direct sum, denoted by $\alpha+\beta$, as the equivalence class of unitary representations of dimension $d_{\alpha}+d_{\beta}$ given by

$$
\left(\begin{array}{cc}
U^{\alpha} & 0 \\
0 & U^{\beta}
\end{array}\right)
$$


and the tensor product, denoted by $\alpha \beta$, is the equivalence class of unitary representations of dimension $d_{\alpha} d_{\beta}$ whose matrix form is given by $U^{\alpha \beta}=U_{13}^{\alpha} U_{23}^{\beta}$.

The character $\chi(\alpha)$ of a finite-dimensional representation $\alpha$ is given by

$$
\chi(\alpha)=\sum_{i=1}^{d_{\alpha}} u_{i i}^{\alpha} .
$$

Note that $\chi(\alpha)$ is independent of the choice of representatives of $\alpha$. Also we have $\|\chi(\alpha)\| \leq d_{\alpha}$, since $\sum_{k=1}^{d_{\alpha}} u_{i k}^{\alpha}\left(u_{i k}^{\alpha}\right)^{*}=1$ for every $1 \leq i \leq d_{\alpha}$. Moreover,

$$
\chi(\alpha+\beta)=\chi(\alpha)+\chi(\beta), \quad \chi(\alpha \beta)=\chi(\alpha) \chi(\beta) \quad \text { and } \quad \chi(\alpha)^{*}=\chi(\bar{\alpha})
$$

for finite-dimensional representations $\alpha$ and $\beta$.

Every representation of a compact quantum group is a direct sum of irreducible representations. For two finite-dimensional representations $\alpha$ and $\beta$, denote by $N_{\alpha, \beta}^{\gamma}$ the number of copies of $\gamma \in \widehat{G}$ in the decomposition of $\alpha \beta$ into a sum of irreducible representations. Hence,

$$
\alpha \beta=\sum_{\gamma \in \widehat{G}} N_{\alpha, \beta}^{\gamma} \gamma .
$$

We have the Frobenius reciprocity law [Woronowicz 1987, Proposition 3.4; Kyed 2008, Example 2.3]

for all $\alpha, \beta, \gamma \in \widehat{G}$.

$$
N_{\alpha, \beta}^{\gamma}=N_{\gamma, \bar{\beta}}^{\alpha}=N_{\bar{\alpha}, \gamma}^{\beta}
$$

Throughout, we assume that $A=C(G)$ is a separable $C^{*}$-algebra, which amounts to saying $\widehat{G}$ is countable.

Definition 2.2 [Kyed 2008, Definition 3.2]. Given two finite subsets $S$ and $F$ of $\widehat{G}$, the boundary of $F$ relative to $S$, denoted by $\partial_{S}(F)$, is defined by

$\partial_{S}(F)=\left\{\alpha \in F \mid N_{\alpha, \gamma}^{\beta}>0\right.$ for some $\left.\gamma \in S, \beta \notin F\right\} \cup\left\{\alpha \notin F \mid N_{\alpha, \gamma}^{\beta}>0\right.$ for some $\left.\gamma \in S, \beta \in F\right\}$.

The weighted cardinality $|F|_{w}$ of a finite subset $F$ of $\widehat{G}$ is given by

$$
|F|_{w}=\sum_{\alpha \in F} d_{\alpha}^{2}
$$

D. Kyed proves a compact quantum group $G$ is coamenable if and only if there exists a Følner sequence in $\widehat{G}$.

Theorem 2.3 (Følner condition for amenable discrete quantum groups [Kyed 2008, Corollary 4.10]). A compact quantum group $G$ is coamenable if and only if there exists a sequence $\left\{F_{n}\right\}_{n=1}^{\infty}$ (a right Folner sequence) of finite subsets of $\widehat{G}$ such that

$$
\lim _{n \rightarrow \infty} \frac{\left|\partial_{S}\left(F_{n}\right)\right|_{w}}{\left|F_{n}\right|_{w}}=0
$$

for every finite nonempty subset $S$ of $\widehat{G}$. 


\section{Mean ergodic theorem for amenable discrete quantum groups}

In this section we prove the generalized mean ergodic theorem.

Theorem 3.1. Let $G$ be a coamenable compact quantum group with counit $\varepsilon$ and $\left\{F_{n}\right\}_{n=1}^{\infty}$ be a right Folner sequence of $\widehat{G}$. For a representation $\pi: A=C(G) \rightarrow B(H)$, we have

$$
\lim _{n \rightarrow \infty} \frac{1}{\left|F_{n}\right|_{w}} \sum_{\alpha \in F_{n}} d_{\alpha} \pi(\chi(\alpha))=P
$$

under the strong operator topology, where $P$ is the orthogonal projection from $\mathrm{H}$ onto

$$
H_{\mathrm{inv}}=\{x \in H \mid \pi(a) x=\varepsilon(a) x \text { for all } a \in A\} .
$$

We divide the proof into two major steps:

Step 1. We show that $H_{\mathrm{inv}}=K$ for $K=\left\{x \in H \mid \pi(\chi(\alpha)) x=d_{\alpha} x\right.$ for all $\left.\alpha \in \widehat{G}\right\}$.

Step 2. The sequence $\left\{\left(1 /\left|F_{n}\right|_{w}\right) \sum_{\alpha \in F_{n}} d_{\alpha} \pi(\chi(\alpha))\right\}_{n=1}^{\infty}$ converges to the projection from $H$ onto $K$. Proof of Step 1 for Theorem 3.1. We proceed via two lemmas:

Lemma 3.2. If a state $\varphi$ on $A=C(G)$ for a compact quantum group $G$ satisfies that $\varphi(\chi(\alpha))=d_{\alpha}$ for all $\alpha \in \widehat{G}$, then $\varphi=\varepsilon$.

Proof. It suffices to show that $\varphi\left(u_{i j}^{\alpha}\right)=\delta_{i j}$ for every $\alpha \in \widehat{G}$ and an arbitrary unitary $U=\left(u_{i j}^{\alpha}\right)_{1 \leq i, j \leq d_{\alpha}} \in \alpha$.

Let $\varphi(U)$ be the matrix $\left(\varphi\left(u_{i j}^{\alpha}\right)\right)$ in $M_{d_{\alpha}}(\mathbb{C})$. Note that $\varphi$ is a state, hence completely positive. By a generalized Schwarz inequality of M. Choi [1974, Corollary 2.8], we have

$$
\varphi(U) \varphi\left(U^{*}\right) \leq \varphi\left(U U^{*}\right)=1 .
$$

Let $\operatorname{Tr}$ be the normalized trace of $M_{d_{\alpha}}(\mathbb{C})$. Since $\varphi(\chi(\alpha))=d_{\alpha}$, we get $\operatorname{Tr}(\varphi(U))=1$. It follows that

$$
\begin{aligned}
0 & \leq \operatorname{Tr}\left((\varphi(U)-1)(\varphi(U)-1)^{*}\right) \\
& =\operatorname{Tr}\left(\varphi(U) \varphi(U)^{*}-\varphi(U)^{*}-\varphi(U)+1\right) \\
& =\operatorname{Tr}\left(\varphi(U) \varphi(U)^{*}\right)-1 \\
& =\operatorname{Tr}\left(\varphi(U) \varphi\left(U^{*}\right)\right)-1 \\
& \leq \operatorname{Tr}\left(\varphi\left(U U^{*}\right)\right)-1=0 .
\end{aligned}
$$

Hence, $\operatorname{Tr}\left((\varphi(U)-1)(\varphi(U)-1)^{*}\right)=0$, which implies that $\varphi(U)=1$. This ends the proof.

Lemma 3.3. Let $\pi: A=C(G) \rightarrow B(H)$ be a representation. Then

$$
H_{\mathrm{inv}}=K=\left\{x \in H \mid \pi(\chi(\alpha)) x=d_{\alpha} x \text { for all } \alpha \in \widehat{G}\right\} .
$$

Proof. Note that $\varepsilon(\chi(\alpha))=d_{\alpha}$ for all $\alpha \in \widehat{G}$ [Woronowicz 1998, Formula (5.11)]. Hence $H_{\text {inv }} \subseteq K$.

To show $K \subseteq H_{\text {inv }}$, we can assume $K \neq 0$ without loss of generality. 
Let $x \in K$ be an arbitrarily chosen unit vector. By Lemma 3.2, the state $\varphi_{x}$ defined by $\varphi_{x}(a)=\langle\pi(a) x, x\rangle$ for all $a \in A$ is $\varepsilon$, since $\varphi_{x}(\chi(\alpha))=d_{\alpha}$ for all $\alpha \in \widehat{G}$.

For every $a \in A$, we have

$$
\begin{aligned}
\|\pi(a) x-\varepsilon(a) x\|^{2} & =\langle\pi(a) x-\varepsilon(a) x, \pi(a) x-\varepsilon(a) x\rangle \\
& =\langle\pi(a) x, \pi(a) x\rangle-\langle\varepsilon(a) x, \pi(a) x\rangle-\langle\pi(a) x, \varepsilon(a) x\rangle+\langle\varepsilon(a) x, \varepsilon(a) x\rangle \\
& =\left\langle\pi\left(a^{*} a\right) x, x\right\rangle-\left\langle\varepsilon(a) \pi\left(a^{*}\right) x, x\right\rangle-\overline{\varepsilon(a)}\langle\pi(a) x, x\rangle+|\varepsilon(a)|^{2} \\
& =\varepsilon\left(a^{*} a\right)-\varepsilon(a) \varepsilon\left(a^{*}\right)-|\varepsilon(a)|^{2}+|\varepsilon(a)|^{2} \\
& =0 .
\end{aligned}
$$

This proves that $K \subseteq H_{\text {inv }}$, and so concludes the proof of Step 1 .

Proof of Step 2 for Theorem 3.1. We start with a lemma:

Lemma 3.4. The orthogonal complement $H_{\mathrm{inv}}^{\perp}$ of $H_{\mathrm{inv}}$ is

$$
V:=\overline{\operatorname{Span}\left\{\pi(\chi(\alpha)) x-d_{\alpha} x \mid \alpha \in \widehat{G}, x \in H\right\}} .
$$

We need the following well-known fact in functional analysis:

Proposition 3.5. Suppose $\left\{T_{j}\right\}_{j \in J}$ is a family of bounded operators on a Hilbert space H. Then the orthogonal complement of $\bigcap_{j \in J} \operatorname{ker} T_{j}$ is

$$
\overline{\operatorname{ran}\left\{T_{j}^{*} \mid j \in J\right\}},
$$

the closed linear span of the ranges $\operatorname{ran} T_{j}^{*}$ of $T_{j}^{*}$ for all $j$ in $J$.

Proof of Lemma 3.4. Consider the family of operators $\left\{\pi(\chi(\alpha))-d_{\alpha}\right\}_{\alpha \in \widehat{G}}$ in $B(H)$. These are self-adjoint operators, since

$$
\left(\pi(\chi(\alpha))-d_{\alpha}\right)^{*}=\pi(\chi(\bar{\alpha}))-d_{\bar{\alpha}}
$$

Applying Proposition 3.5 to $\left\{\pi(\chi(\alpha))-d_{\alpha}\right\}_{\alpha \in \widehat{G}}$ gives the proof.

Now we are ready to finish the proof of Theorem 3.1.

For every $x \in H_{\text {inv }}$ and all $n$, we have

$$
\frac{1}{\left|F_{n}\right|_{w}} \sum_{\alpha \in F_{n}} d_{\alpha} \pi(\chi(\alpha)) x=\frac{1}{\left|F_{n}\right|_{w}} \sum_{\alpha \in F_{n}} d_{\alpha}^{2} x=x .
$$

Next we show that

$$
\frac{1}{\left|F_{n}\right|_{w}} \sum_{\alpha \in F_{n}} d_{\alpha} \pi(\chi(\alpha)) z \rightarrow 0
$$

for all $z \in V$ as $n \rightarrow \infty$. By Lemma 3.4, we only need to prove it for $z$ of the form $\pi(\chi(\gamma)) y-d_{\gamma} y$ for every $y \in H$ and $\gamma \in \widehat{G}$. 
For every $y \in H$ and $\gamma \in \widehat{G}$, we have

$$
\begin{aligned}
& \lim _{n \rightarrow \infty} \frac{1}{\left|F_{n}\right|_{w}} \sum_{\alpha \in F_{n}} d_{\alpha} \pi(\chi(\alpha))\left(\pi(\chi(\gamma)) y-d_{\gamma} y\right) \\
& =\lim _{n \rightarrow \infty} \frac{1}{\left|F_{n}\right|_{w}}\left(\sum_{\alpha \in F_{n} \backslash \partial_{\gamma} F_{n}}+\sum_{\alpha \in F_{n} \cap \partial_{\gamma} F_{n}}\right) d_{\alpha} \pi(\chi(\alpha) \chi(\gamma)) y-d_{\alpha} d_{\gamma} \pi(\chi(\alpha)) y \\
& =\lim _{n \rightarrow \infty} \frac{1}{\left|F_{n}\right|_{w}} \sum_{\alpha \in F_{n} \backslash \partial_{\gamma} F_{n}} d_{\alpha} \pi(\chi(\alpha \gamma)) y-d_{\alpha} d_{\gamma} \pi(\chi(\alpha)) y \quad\left(\alpha \gamma=\sum_{\beta \in F_{n}} N_{\alpha, \gamma}^{\beta} \beta \text { when } \alpha \in F_{n} \backslash \partial_{\gamma} F_{n}\right) \\
& =\lim _{n \rightarrow \infty} \frac{1}{\left|F_{n}\right|_{w}}\left(\sum_{\alpha \in F_{n} \backslash \partial_{\gamma} F_{n}} \sum_{\beta \in F_{n}} d_{\alpha} N_{\alpha, \gamma}^{\beta} \pi(\chi(\beta)) y-\sum_{\alpha \in F_{n} \backslash \partial_{\gamma} F_{n}} d_{\alpha} d_{\gamma} \pi(\chi(\alpha)) y\right) \\
& =\lim _{n \rightarrow \infty} \frac{1}{\left|F_{n}\right|_{w}}\left(\sum_{\alpha \in F_{n} \backslash \partial_{\gamma} F_{n}} \sum_{\beta \in F_{n}} d_{\alpha} N_{\beta, \bar{\gamma}}^{\alpha} \pi(\chi(\beta)) y-\sum_{\alpha \in F_{n} \backslash \partial_{\gamma} F_{n}} d_{\alpha} d_{\bar{\gamma}} \pi(\chi(\alpha)) y\right) \\
& =\lim _{n \rightarrow \infty} \frac{1}{\left|F_{n}\right|_{w}}\left(\sum_{\alpha \in F_{n} \backslash \partial_{\gamma} F_{n}} \sum_{\beta \in F_{n}} d_{\alpha} N_{\beta, \gamma}^{\alpha} \pi(\chi(\beta)) y-\sum_{\alpha \in F_{n} \backslash \partial_{\gamma} F_{n}}\left[\sum_{\beta \in F_{n}}+\sum_{\beta \notin F_{n}}\right] N_{\alpha, \bar{\gamma}}^{\beta} d_{\beta} \pi(\chi(\alpha)) y\right)
\end{aligned}
$$

(exchange $\alpha$ and $\beta$ in the second term)

$=\lim _{n \rightarrow \infty} \frac{1}{\left|F_{n}\right|_{w}}\left(\sum_{\alpha \in F_{n} \backslash \partial_{\gamma} F_{n}} \sum_{\beta \in F_{n}} d_{\alpha} N_{\beta, \bar{\gamma}}^{\alpha} \pi(\chi(\beta)) y-\sum_{\beta \in F_{n} \backslash \partial_{\gamma} F_{n}}\left[\sum_{\alpha \in F_{n}}+\sum_{\alpha \notin F_{n}}\right] N_{\beta, \bar{\gamma}}^{\alpha} d_{\alpha} \pi(\chi(\beta)) y\right)$

(common terms are canceled)

$$
\begin{aligned}
=\lim _{n \rightarrow \infty} \frac{1}{\left|F_{n}\right|_{w}}\left(\sum_{\alpha \in F_{n} \backslash \partial_{\gamma} F_{n}} \sum_{\beta \in F_{n} \cap \partial_{\gamma} F_{n}} d_{\alpha} N_{\beta, \bar{\gamma}}^{\alpha} \pi(\chi(\beta)) y\right. & \\
& \left.\quad-\sum_{\beta \in F_{n} \backslash \partial_{\gamma} F_{n}} \sum_{\alpha \in F_{n} \cap \partial_{\gamma} F_{n}} N_{\beta, \bar{\gamma}}^{\alpha} d_{\beta} \pi(\chi(\beta)) y-\sum_{\beta \in F_{n} \backslash \partial_{\gamma} F_{n}} \sum_{\alpha \notin F_{n}} N_{\beta, \bar{\gamma}}^{\alpha} d_{\alpha} \pi(\chi(\beta)) y\right)
\end{aligned}
$$

$=0$.

Note that the last equality above holds since, by Theorem 2.3, we have the following:

(1) $\frac{1}{\left|F_{n}\right|_{w}}\left\|\sum_{\alpha \in F_{n} \backslash \partial_{\gamma} F_{n}} \sum_{\beta \in F_{n} \cap \partial_{\gamma} F_{n}} d_{\alpha} N_{\beta, \bar{\gamma}}^{\alpha} \pi(\chi(\beta)) y\right\| \leq \frac{1}{\left|F_{n}\right|_{w}} \sum_{\beta \in F_{n} \cap \partial_{\gamma} F_{n}} \sum_{\alpha \in F_{n}} d_{\alpha} N_{\beta, \bar{\gamma}}^{\alpha} d_{\beta}\|y\|$

$$
\leq \frac{1}{\left|F_{n}\right|_{w}} \sum_{\beta \in F_{n} \cap \partial_{\gamma} F_{n}} d_{\beta}^{2} d_{\bar{\gamma}}\|y\| \rightarrow 0
$$

(2) $\frac{1}{\left|F_{n}\right|_{w}}\left\|\sum_{\beta \in F_{n} \backslash \partial_{\gamma} F_{n}} \sum_{\alpha \in F_{n} \cap \partial_{\gamma} F_{n}} N_{\beta, \bar{\gamma}}^{\alpha} d_{\alpha} \pi(\chi(\beta)) y\right\| \leq \frac{1}{\left|F_{n}\right|_{w}} \sum_{\beta \in F_{n} \backslash \partial_{\gamma} F_{n}} \sum_{\alpha \in F_{n} \cap \partial_{\gamma} F_{n}} N_{\beta, \bar{\gamma}}^{\alpha} d_{\alpha} d_{\beta}\|y\|$

$$
\begin{aligned}
& =\frac{1}{\left|F_{n}\right|_{w}} \sum_{\beta \in F_{n} \backslash \partial_{\gamma} F_{n}} \sum_{\alpha \in F_{n} \cap \partial_{\gamma} F_{n}} N_{\alpha, \gamma}^{\beta} d_{\alpha} d_{\beta}\|y\| \\
& \leq \frac{1}{\left|F_{n}\right|_{w}} \sum_{\alpha \in F_{n} \cap \partial_{\gamma} F_{n}} d_{\alpha}^{2} d_{\gamma}\|y\| \rightarrow 0 ;
\end{aligned}
$$




$$
\text { (3) } \begin{aligned}
\frac{1}{\left|F_{n}\right|_{w}}\left\|\sum_{\beta \in F_{n} \backslash \partial_{\gamma} F_{n}} \sum_{\alpha \notin F_{n}} N_{\beta, \bar{\gamma}}^{\alpha} d_{\alpha} \pi(\chi(\beta)) y\right\| & \leq \frac{1}{\left|F_{n}\right|_{w}} \sum_{\beta \in F_{n} \backslash \partial_{\gamma} F_{n}} \sum_{\alpha \notin F_{n}} N_{\beta, \bar{\gamma}}^{\alpha} d_{\alpha} d_{\beta}\|y\| \\
& =\frac{1}{\left|F_{n}\right|_{w}} \sum_{\beta \in F_{n} \backslash \partial_{\gamma} F_{n}} \sum_{\alpha \notin F_{n}, N_{\beta, \bar{\gamma}}^{\alpha}>0} N_{\beta, \bar{\gamma}}^{\alpha} d_{\alpha} d_{\beta}\|y\| \\
& \leq \frac{1}{\left|F_{n}\right|_{w}} \sum_{\beta \in \partial_{\bar{\gamma}} F_{n}} \sum_{\alpha \in \widehat{G}} N_{\beta, \bar{\gamma}}^{\alpha} d_{\alpha} d_{\beta}\|y\| \\
& =\frac{1}{\left|F_{n}\right|_{w}} \sum_{\beta \in \partial_{\bar{\gamma}} F_{n}} d_{\beta}^{2} d_{\bar{\gamma}}\|y\| \rightarrow 0 \text { as } n \rightarrow \infty .
\end{aligned}
$$

This completes proof of Step 2 and therefore of Theorem 3.1.

For a representation $\pi: B \rightarrow B(H)$ of a unital $C^{*}$-algebra $B$, define the commutant $\pi(B)^{\prime}$ of $\pi(B)$ by

$$
\pi(B)^{\prime}=\{T \in B(H) \mid T \pi(b)=\pi(b) T \text { for all } b \in B\} .
$$

Corollary 3.6. In the setting of Theorem 3.1, the projection $P$ is in $\pi(A)^{\prime} \cap \overline{\pi(A)}^{\mathrm{SOT}}$.

Proof. The left-hand side of (3-1) is in $\overline{\pi(A)}^{\text {SOT }}$; hence, so is $P$. Moreover, for all $x, y \in H$ and $a \in A$, we have

$$
\langle\pi(a) P x, y\rangle=\varepsilon(a)\langle P x, y\rangle
$$

and

$$
\langle P \pi(a) x, y\rangle=\langle\pi(a) x, P y\rangle=\left\langle x, \pi\left(a^{*}\right) P y\right\rangle=\left\langle x, \varepsilon\left(a^{*}\right) P y\right\rangle=\varepsilon(a)\langle P x, y\rangle .
$$

This proves $P \in \pi(A)^{\prime}$.

As a consequence, we have the following:

Corollary 3.7. Assume that $\varphi$ is a pure state on $A=C(G)$ for a coamenable compact quantum group $G$ and $\left\{F_{n}\right\}_{n=1}^{\infty}$ is a right Folner sequence of $\widehat{G}$. Then

$$
\lim _{n \rightarrow \infty} \frac{1}{\left|F_{n}\right|_{w}} \sum_{\alpha \in F_{n}} d_{\alpha} \varphi(\chi(\alpha))= \begin{cases}1 & \text { if } \varphi=\varepsilon \\ 0 & \text { if } \varphi \neq \varepsilon\end{cases}
$$

Proof. When $\varphi=\varepsilon$, we have $\varepsilon(\chi(\alpha))=d_{\alpha}$ for all $\alpha \in \widehat{G}$ [Woronowicz 1998, Formula (5.11)]. Hence,

$$
\lim _{n \rightarrow \infty} \frac{1}{\left|F_{n}\right|_{w}} \sum_{\alpha \in F_{n}} d_{\alpha} \varepsilon(\chi(\alpha))=1
$$

Suppose $\varphi \neq \varepsilon$.

Consider the GNS representation $\pi_{\varphi}: A \rightarrow B\left(L^{2}(A, \varphi)\right)$. We have

$$
\lim _{n \rightarrow \infty} \frac{1}{\left|F_{n}\right|_{w}} \sum_{\alpha \in F_{n}} d_{\alpha} \varphi(\chi(\alpha))=\lim _{n \rightarrow \infty} \frac{1}{\left|F_{n}\right|_{w}} \sum_{\alpha \in F_{n}} d_{\alpha}\left\langle\pi_{\varphi}(\chi(\alpha))(\hat{1}), \hat{1}\right\rangle=\langle P(\hat{1}), \hat{1}\rangle .
$$

Hence, $\lim _{n \rightarrow \infty}\left(1 /\left|F_{n}\right|_{w}\right) \sum_{\alpha \in F_{n}} d_{\alpha} \varphi(\chi(\alpha)) \neq 0$ if and only if $P(\hat{1}) \neq 0$.

To prove $\lim _{n \rightarrow \infty}\left(1 /\left|F_{n}\right|_{w}\right) \sum_{\alpha \in F_{n}} d_{\alpha} \varphi(\chi(\alpha))=0$ for $\varphi \neq \varepsilon$, it suffices to prove $P(\hat{1})=0$. 
Suppose $P(\hat{1}) \neq 0$. Then $H_{\text {inv }} \neq 0$. By Corollary 3.6, the space $H_{\text {inv }}$ is an invariant subspace of $L^{2}(A, \varphi)$. Note that $\pi_{\varphi}$ is irreducible since $\varphi$ is a pure state. Hence $H_{\text {inv }}=L^{2}(A, \varphi)$. In particular, $\hat{1} \in H_{\text {inv }}$. Thus, for all $a \in A$, we have $\pi_{\varphi}(a)(\hat{1})=\varepsilon(a) \hat{1}$. It follows that

$$
\varphi(a)=\left\langle\pi_{\varphi}(a)(\hat{1}), \hat{1}\right\rangle=\langle\varepsilon(a) \hat{1}, \hat{1}\rangle=\varepsilon(a)
$$

for all $a \in A$, which contradicts that $\varphi \neq \varepsilon$.

\section{A Wiener-type theorem for compact metrizable groups}

In this section, we prove the following Wiener-type theorem:

Theorem 4.1. Let $G$ be a compact metrizable group. Given $y$ in $G$ and a right Følner sequence $\left\{F_{n}\right\}_{n=1}^{\infty}$ of $\widehat{G}$, for a finite Borel measure $\mu$ on $G$ one has

$\lim _{n \rightarrow \infty} \frac{1}{\left|F_{n}\right|_{w}} \sum_{\alpha \in F_{n}} d_{\alpha} \sum_{1 \leq i, j \leq d_{\alpha}} \mu\left(u_{i j}^{\alpha}\right) \overline{u_{i j}^{\alpha}(y)}=\mu\{y\} \quad$ and $\quad \lim _{n \rightarrow \infty} \frac{1}{\left|F_{n}\right|_{w}} \sum_{\alpha \in F_{n}} d_{\alpha} \sum_{1 \leq i, j \leq d_{\alpha}}\left|\mu\left(u_{i j}^{\alpha}\right)\right|^{2}=\sum_{x \in G} \mu\{x\}^{2}$.

Hence, $\mu$ is continuous if and only if

$$
\lim _{n \rightarrow \infty} \frac{1}{\left|F_{n}\right|_{w}} \sum_{\alpha \in F_{n}} d_{\alpha} \sum_{1 \leq i, j \leq d_{\alpha}}\left|\mu\left(u_{i j}^{\alpha}\right)\right|^{2}=0 .
$$

Here $\left(u_{i j}^{\alpha}\right)_{1 \leq i, j \leq d_{\alpha}} \in M_{d_{\alpha}}(C(G))$ stands for a unitary matrix presenting $\alpha \in \widehat{G}$.

From now on $G$ stands for a compact metrizable group. When thinking of $G$ as a compact quantum group, the coproduct

$$
\Delta: C(G) \rightarrow C(G) \otimes C(G)
$$

is given by $\Delta(f)(x, y)=f(x y)$, the coinverse $\kappa: C(G) \rightarrow C(G)$ is given by $\kappa(f)(x)=f\left(x^{-1}\right)$ and the counit $\varepsilon: C(G) \rightarrow \mathbb{C}$ is given by $\varepsilon(f)=f\left(e_{G}\right)$ for all $f \in C(G)$ and $x, y \in G$. Here, $e_{G}$ is the neutral element of $G$.

Definition 4.2. Given a finite Borel measure $\mu$ on $G$, the conjugate $\bar{\mu}$ of $\mu$ is defined by

$$
\bar{\mu}(f)=\int_{G} f\left(x^{-1}\right) d \mu(x)=\mu(\kappa(f))
$$

for all $f \in C(G)$, and $\bar{\mu}$ is also a finite Borel measure on $G$. In other words, $\bar{\mu}(E)=\mu\left(E^{-1}\right)$ for every Borel subset $E$ of $G$.

For $x \in G$, use $\delta_{x}$ to denote the Dirac measure at $x$.

The convolution $\mu * v$ of two finite Borel measures $\mu$ and $v$ on $G$ is defined by

$$
\mu * \nu(f)=(\mu \otimes \nu) \Delta(f)=\int_{G} \int_{G} f(x y) d \mu(x) d v(y)
$$

for all $f \in C(G)$. For every Borel subset $E$ of $G$, we have

$$
\mu * \nu(E)=\int_{G} v\left(x^{-1} E\right) d \mu(x)=\int_{G} \mu\left(E y^{-1}\right) d \nu(y) .
$$


If either $\mu$ or $v$ is continuous, then so is $\mu * v$.

We can write a finite Borel measure $\mu$ on $G$ as $\mu=\sum_{i} \lambda_{i} \delta_{x_{i}}+\mu_{C}$ for every atom $x_{i}$ with $\mu\left\{x_{i}\right\}=\lambda_{i}$ and a finite continuous Borel measure $\mu_{C}$.

Lemma 4.3. Let $\mu$ be a finite Borel measure on $G$ and $\left\{F_{n}\right\}_{n=1}^{\infty}$ be a right $F \phi l n e r$ sequence of $\widehat{G}$. Then

$$
\lim _{n \rightarrow \infty} \frac{1}{\left|F_{n}\right|_{w}} \sum_{\alpha \in F_{n}} d_{\alpha} \mu(\chi(\alpha))=\mu\left\{e_{G}\right\}
$$

Proof. By Corollary 3.7, the sequence $\left\{\left(1 /\left|F_{n}\right|_{w}\right) \sum_{\alpha \in F_{n}} d_{\alpha} \chi(\alpha)(x)\right\} \subseteq C(G)$ converges pointwise to $1_{e_{G}}$ (the characteristic function of $\left\{e_{G}\right\}$ ). The terms of the sequence are bounded by 1 for all $x \in G$; hence, by Lebesgue's dominated convergence theorem [Rudin 1987, Theorem 1.34], we have

$$
\begin{aligned}
\lim _{n \rightarrow \infty} \frac{1}{\left|F_{n}\right|_{w}} \sum_{\alpha \in F_{n}} d_{\alpha} \mu(\chi(\alpha)) & =\lim _{n \rightarrow \infty} \int_{G} \frac{1}{\left|F_{n}\right|_{w}} \sum_{\alpha \in F_{n}} d_{\alpha} \chi(\alpha)(x) d \mu(x) \\
& =\int_{G} \lim _{n \rightarrow \infty} \frac{1}{\left|F_{n}\right|_{w}} \sum_{\alpha \in F_{n}} d_{\alpha} \chi(\alpha)(x) d \mu(x) \\
& =\int_{G} 1_{e_{G}} d \mu=\mu\left\{e_{G}\right\} .
\end{aligned}
$$

Proof of Theorem 4.1. Given a finite Borel measure $\mu$ on $G$ and $y \in G$, consider the measure $\mu * \delta_{y^{-1}}$. By Lemma 4.3, we have

$$
\lim _{n \rightarrow \infty} \frac{1}{\left|F_{n}\right|_{w}} \sum_{\alpha \in F_{n}} d_{\alpha} \mu * \delta_{y^{-1}}(\chi(\alpha))=\mu * \delta_{y^{-1}}\left\{e_{G}\right\}
$$

Note that

$$
\begin{aligned}
\mu * \delta_{y^{-1}}(\chi(\alpha)) & =\int_{G} \int_{G} \chi(\alpha)(x z) d \mu(x) d \delta_{y^{-1}}(z) \\
& =\int_{G} \chi(\alpha)\left(x y^{-1}\right) d \mu(x) \\
& =\int_{G} \sum_{1 \leq i \leq d_{\alpha}} u_{i i}^{\alpha}\left(x y^{-1}\right) d \mu(x) \\
& =\int_{G} \sum_{1 \leq i \leq d_{\alpha}} \sum_{1 \leq j \leq d_{\alpha}} u_{i j}^{\alpha}(x) u_{j i}^{\alpha}\left(y^{-1}\right) d \mu(x) \\
& =\int_{G} \sum_{1 \leq i \leq d_{\alpha}} \sum_{1 \leq j \leq d_{\alpha}} u_{i j}^{\alpha}(x) \overline{u_{i j}^{\alpha}(y)} d \mu(x) .
\end{aligned}
$$

Moreover,

$$
\mu * \delta_{y^{-1}}\left\{e_{G}\right\}=\int_{G} \int_{G} 1_{e_{G}}(x z) d \mu(x) d \delta_{y^{-1}}(z)=\int_{G} 1_{e_{G}}\left(x y^{-1}\right) d \mu(x)=\mu\{y\} .
$$

This completes the proof of the first part. 
Applying Lemma 4.3 to $\mu * \bar{\mu}$, we have

$$
\lim _{n \rightarrow \infty} \frac{1}{\left|F_{n}\right|_{w}} \sum_{\alpha \in F_{n}} d_{\alpha} \mu * \bar{\mu}(\chi(\alpha))=\mu * \bar{\mu}\left\{e_{G}\right\}
$$

Since $\mu=\sum_{x_{i} \text { atoms }} \lambda_{i} \delta_{x_{i}}+\mu_{C}$ with $\lambda_{i}=\mu\left\{x_{i}\right\}$ and $\mu_{C}$ a finite continuous Borel measure, we have

Hence,

$$
\bar{\mu}=\sum_{x_{i} \text { atoms }} \lambda_{i} \bar{\delta}_{x_{i}}+\overline{\mu_{C}}=\sum_{x_{i} \text { atoms }} \lambda_{i} \delta_{x_{i}^{-1}}+\overline{\mu_{C}} .
$$

$$
\mu * \bar{\mu}=\sum_{i} \sum_{j} \lambda_{i} \lambda_{j} \delta_{x_{i}} * \delta_{x_{j}^{-1}}+\sum_{i} \lambda_{i} \delta_{x_{i}} * \overline{\mu_{C}}+\sum_{j} \lambda_{j} \mu_{C} * \delta_{x_{j}^{-1}}+\mu_{C} * \overline{\mu_{C}} .
$$

Note that $\sum_{i} \lambda_{i} \delta_{x_{i}} * \overline{\mu_{C}}+\sum_{j} \lambda_{j} \mu_{C} * \delta_{x_{j}^{-1}}+\mu_{C} * \overline{\mu_{C}}$ is a finite continuous measure and

It follows that

$$
\sum_{i, j} \lambda_{i} \lambda_{j} \delta_{x_{i}} * \delta_{x_{j}^{-1}}=\sum_{i, j} \lambda_{i} \lambda_{j} \delta_{x_{i} x_{j}^{-1}}
$$

$$
\mu * \bar{\mu}\left\{e_{G}\right\}=\sum_{x_{i} \text { atoms }} \lambda_{i}^{2}=\sum_{x_{i} \text { atoms }} \mu\left\{x_{i}\right\}^{2}=\sum_{x \in G} \mu\{x\}^{2} .
$$

On the other hand,

$$
\begin{aligned}
\mu * \bar{\mu}(\chi(\alpha)) & =\int_{G} \int_{G} \chi(\alpha)(x y) d \mu(x) d \bar{\mu}(y) \\
& =\int_{G} \int_{G} \chi(\alpha)\left(x y^{-1}\right) d \mu(x) d \mu(y) \\
& =\int_{G} \int_{G} \sum_{1 \leq i \leq d_{\alpha}} u_{i i}^{\alpha}\left(x y^{-1}\right) d \mu(x) d \mu(y) \\
& =\int_{G} \int_{G} \sum_{1 \leq i \leq d_{\alpha}} \sum_{1 \leq j \leq d_{\alpha}} u_{i j}^{\alpha}(x) u_{j i}^{\alpha}\left(y^{-1}\right) d \mu(x) d \mu(y) \\
& =\sum_{1 \leq i \leq d_{\alpha}} \sum_{1 \leq j \leq d_{\alpha}} \int_{G} u_{i j}^{\alpha}(x) d \mu(x) \int_{G} \overline{u_{i j}^{\alpha}(y)} d \mu(y) \\
& =\sum_{1 \leq i, j \leq d_{\alpha}}\left|\mu\left(u_{i j}^{\alpha}\right)\right|^{2} .
\end{aligned}
$$

This ends the proof of the first part, and the second follows immediately.

\section{Acknowledgements}

The paper was finished when I was a postdoctoral fellow from June 2013 to January 2016, supported by ERC Advanced Grant No. 267079. I express my gratitude to my mentor Joachim Cuntz. I thank Martijn Caspers for pointing out the reference [Kyed 2008] to me, which motivates the article. I am grateful to Hanfeng Li and Shuzhou Wang for their comments. I thank Ami Viselter for reminding me of some 
preceding works. Last but not least, I thank the anonymous referee and the editor for their comments and suggestions, which greatly improve the readability of the article.

\section{References}

[Anoussis and Bisbas 2000] M. Anoussis and A. Bisbas, "Continuous measures on compact Lie groups", Ann. Inst. Fourier (Grenoble) 50:4 (2000), 1277-1296. MR 1799746 Zbl 0969.43001

[Bédos et al. 2001] E. Bédos, G. J. Murphy, and L. Tuset, "Co-amenability of compact quantum groups", J. Geom. Phys. 40:2 (2001), 130-153. MR 1862084 Zbl 1011.46056

[Björklund and Fish 2009] M. Björklund and A. Fish, "Continuous measures on homogenous spaces", Ann. Inst. Fourier (Grenoble) 59:6 (2009), 2169-2174. MR 2640917 Zbl 1194.60009

[Choi 1974] M. D. Choi, "A Schwarz inequality for positive linear maps on $C^{*}$-algebras", Illinois J. Math. 18:4 (1974), 565-574. MR 0355615 Zbl 0293.46043

[Duvenhage 2008] R. Duvenhage, "A mean ergodic theorem for actions of amenable quantum groups", Bull. Aust. Math. Soc. 78:1 (2008), 87-95. MR 2458300 Zbl 1160.46043

[Glasner 2003] E. Glasner, Ergodic theory via joinings, Mathematical Surveys and Monographs 101, American Mathematical Society, Providence, RI, 2003. MR 1958753 Zbl 1038.37002

[Katznelson 2004] Y. Katznelson, An introduction to harmonic analysis, 3rd ed., Cambridge University Press, 2004. MR 2039503 Zbl 1055.43001

[Kyed 2008] D. Kyed, " $L^{2}$-Betti numbers of coamenable quantum groups”, Münster J. Math. 1 (2008), 143-179. MR 2502497 Zbl 1195.46073

[Ruan 1996] Z.-J. Ruan, “Amenability of Hopf von Neumann algebras and Kac algebras”, J. Funct. Anal. 139:2 (1996), 466-499. MR 1402773 Zbl 0896.46041

[Rudin 1987] W. Rudin, Real and complex analysis, 3rd ed., McGraw-Hill, New York, 1987. MR 924157 Zbl 0925.00005

[Runde and Viselter 2014] V. Runde and A. Viselter, "Ergodic theory for quantum semigroups", J. Lond. Math. Soc. (2) 89:3 (2014), 941-959. MR 3217657

[Taylor 1981] M. E. Taylor, Pseudodifferential operators, Princeton Mathematical Series 34, Princeton University Press, 1981. MR 618463 Zbl 0453.47026

[Tomatsu 2006] R. Tomatsu, "A paving theorem for amenable discrete Kac algebras", Internat. J. Math. 17:8 (2006), 905-919. MR 2261640 Zbl 1115.46057

[Wiener 1933] N. Wiener, The Fourier integral and certain of its applications, Dover, New York, 1933. MR 983891 Zbl 0006.05401

[Woronowicz 1987] S. L. Woronowicz, "Compact matrix pseudogroups”, Comm. Math. Phys. 111 (1987), 613-665. MR 901157 Zbl 0627.58034

[Woronowicz 1998] S. L. Woronowicz, “Compact quantum groups”, pp. 845-884 in Symétries quantiques (Les Houches, 1995), edited by A. Connes et al., North-Holland, Amsterdam, 1998. MR 1616348 Zbl 0997.46045

Received 10 Nov 2015. Revised 3 Feb 2016. Accepted 11 Mar 2016.

HUICHI HUANG: huichi-huang@hotmail .com

College of Mathematics and Statistics, Chongqing University, Chongqing, 401331, China 


\title{
Analysis \& PDE
}

msp.org/apde

\section{EDITORS}

\author{
EDITOR-IN-CHIEF \\ Patrick Gérard \\ patrick.gerard@math.u-psud.fr \\ Université Paris Sud XI \\ Orsay, France
}

BOARD OF EDITORS

Nicolas Burq Université Paris-Sud 11, France nicolas.burq@math.u-psud.fr

Massimiliano Berti Scuola Intern. Sup. di Studi Avanzati, Italy berti@sissa.it

Sun-Yung Alice Chang Princeton University, USA chang@math.princeton.edu

Michael Christ University of California, Berkeley, USA mchrist@math.berkeley.edu

Charles Fefferman Princeton University, USA cf@math.princeton.edu

Ursula Hamenstaedt Universität Bonn, Germany ursula@math.uni-bonn.de

Vaughan Jones U.C. Berkeley \& Vanderbilt University vaughan.f.jones@vanderbilt.edu

Vadim Kaloshin University of Maryland, USA vadim.kaloshin@gmail.com

Herbert Koch Universität Bonn, Germany koch@math.uni-bonn.de

Izabella Laba University of British Columbia, Canada ilaba@math.ubc.ca

Gilles Lebeau Université de Nice Sophia Antipolis, France lebeau@unice.fr

László Lempert Purdue University, USA lempert@math.purdue.edu

Richard B. Melrose Massachussets Inst. of Tech., USA rbm@math.mit.edu

Frank Merle Université de Cergy-Pontoise, France Frank.Merle@u-cergy.fr

William Minicozzi II Johns Hopkins University, USA minicozz@math.jhu.edu

Clément Mouhot Cambridge University, UK c.mouhot@dpmms.cam.ac.uk
Werner Müller

Yuval Peres

Gilles Pisier

Tristan Rivière

Igor Rodnianski

Wilhelm Schlag

Sylvia Serfaty

Yum-Tong Siu

Terence Tao

Michael E. Taylor

Gunther Uhlmann

András Vasy

Dan Virgil Voiculescu

Steven Zelditch

Maciej Zworski
Universität Bonn, Germany mueller@math.uni-bonn.de

University of California, Berkeley, USA peres@stat.berkeley.edu

Texas A\&M University, and Paris 6 pisier@math.tamu.edu

ETH, Switzerland riviere@math.ethz.ch Princeton University, USA irod@math.princeton.edu University of Chicago, USA schlag@math.uchicago.edu New York University, USA serfaty@cims.nyu.edu Harvard University, USA siu@math.harvard.edu University of California, Los Angeles, USA tao@math.ucla.edu Univ. of North Carolina, Chapel Hill, USA met@math.unc.edu

University of Washington, USA gunther@math.washington.edu

Stanford University, USA andras@math.stanford.edu

University of California, Berkeley, USA dvv@math.berkeley.edu

Northwestern University, USA zelditch@math.northwestern.edu

University of California, Berkeley, USA zworski@math.berkeley.edu

PRODUCTION

production@msp.org

Silvio Levy, Scientific Editor

See inside back cover or msp.org/apde for submission instructions.

The subscription price for 2016 is US \$235/year for the electronic version, and \$430/year (+\$55, if shipping outside the US) for print and electronic. Subscriptions, requests for back issues from the last three years and changes of subscribers address should be sent to MSP.

Analysis \& PDE (ISSN 1948-206X electronic, 2157-5045 printed) at Mathematical Sciences Publishers, 798 Evans Hall \#3840, c/o University of California, Berkeley, CA 94720-3840, is published continuously online. Periodical rate postage paid at Berkeley, CA 94704, and additional mailing offices.

APDE peer review and production are managed by EditFlow ${ }^{\circledR}$ from MSP.

PUBLISHED BY

- mathematical sciences publishers

nonprofit scientific publishing

http://msp.org/

(C) 2016 Mathematical Sciences Publishers 


\section{ANALYSIS \& PDE \\ Volume $9 \quad$ No. $4 \quad 2016$}

Peierls substitution for magnetic Bloch bands

Silvia Freund and Stefan TEUfEL

Dispersive estimates in $\mathbb{R}^{3}$ with threshold eigenstates and resonances

MARIUS BECEANU

Interior nodal sets of Steklov eigenfunctions on surfaces

859

JIUYI ZHU

Some counterexamples to Sobolev regularity for degenerate Monge-Ampère equations

881 CONNOR MOONEY

Mean ergodic theorem for amenable discrete quantum groups and a Wiener-type theorem for

893 compact metrizable groups

HUICHI HUANG

Resonance free regions for nontrapping manifolds with cusps

KIRIL DATCHEV

Characterizing regularity of domains via the Riesz transforms on their boundaries

Dorina Mitrea, Marius Mitrea and JoAn VERdERA 